\title{
STEADY MOTION OF AN INSULATING RIGID FLAT-ENDED PUNCH OVER A THERMALLY CONDUCTING HALF-PLANE*
}

\author{
D. A. HILLS ${ }^{\dagger}$ and J. R. BARBER
}

Department of Mechanical Engineering and Applied Mechanics, University of Michigan, Ann Arbor, MI 48109 (U.S.A.)

(Received June 19, 1984; accepted October 1, 1984)

\section{Summary}

An insulated, rigid, flatended punch moving steadily over the surface of a half-plane is considered. It is assumed that the motion has been sustained for a sufficiently long period for a steady thermal state to have been achieved, the source of heat being the energy dissipated at the interface due to Coulomb friction. The effect of thermal distortion on the contact pressure distribution is found and the temperature profile under the punch is given. A closed-form solution is found for the case of large Peclet number.

\section{Introduction}

In many sliding contact problems, the heat generated by friction produces significant thermoelastic distortion of the contacting surfaces and hence affects the contact pressure distribution. The action of non-metallic brake shoes against steel brake drums is a particular case where this phenomenon is pronounced. Closed-form solutions to this type of thermoelastic contact problem are rare, but the integral equation approach presented here permits a concise formulation and efficient numerical treatment. It is made possible by the recently published Green's function for a line heat source moving steadily over a half-plane [1]. A necessary restriction is that the whole of the heat generated flows into the half-plane without any loss to the surroundings. Thus, the treatment is of greatest applicability where the halfplane is metallic, and hence a good conductor, whilst the punch is of poor conductivity. The brake shoe-drum arrangement mentioned above typifies this configuration.

\footnotetext{
* Paper presented at the Third Workshop on Thermomechanical Effects in Sliding Systems, Hanover, NH, U.S.A., June 18 - 20, 1984.

†Permanent address: Department of Engineering Science, Oxford University, Parks Road, Oxford OX1 3PJ, Gt. Britain.
} 


\section{Formulation}

The geometry of the problem to be considered is illustrated in Fig. 1. A non-conducting flat-ended rigid punch of semiwidth $a$ is pressed against the elastic half-plane $y<0$ by a normal force $P$ and slides to the right at a constant speed $V$. Coulomb friction is assumed to occur at the interface, giving tangential tractions which are everywhere proportional to the normal tractions $p(x)$ through a coefficient of friction $f$. Heat is therefore generated at the interface owing to friction and is conducted into the half-plane.

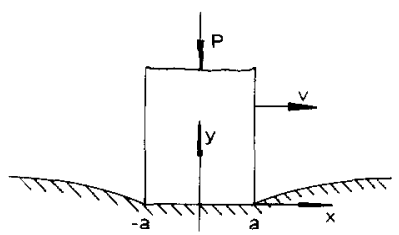

Fig. 1. Geometry of the sliding punch.

The contact problem is conveniently defined in a coordinate system $(x, y)$ which moves with respect to the punch. We treat the traction distribution $p(x)$ under the punch in the region $-a<x<a$ as the unknown and find it from the requirement that the sum of the elastic displacements due to the normal and tangential tractions and of the thermoelastic displacements due to the heat input should be constant under the punch if contact is to be maintained.

The normal displacement at the point $(x, 0)$ due to a line heat source of strength $q$ at the point $(\xi, 0)$ is given in ref. 1 as

$$
\begin{array}{ll}
u_{\text {th }}=\frac{2 \alpha q(1+\nu)}{\rho c V} \exp \left\{-\frac{V(x-\xi)}{2 k}\right\} I_{0}\left\{\frac{V(x-\xi)}{2 k}\right\} \quad x>\xi \\
u_{\text {th }}=\frac{2 \alpha q(1+\nu)}{\rho c V} \quad x<\xi
\end{array}
$$

where $\nu, k, \rho, c$ and $\alpha$ are respectively Poisson's ratio, the thermal diffusivity, the density, the specific heat and the coefficient of thermal expansion for the material of the half-plane. The source has been moving indefinitely at a constant speed $V(V>0)$ and $I_{0}$ is a modified Bessel function.

Equations (1) may be differentiated with respect to $x$, giving

$$
\begin{aligned}
\frac{\mathrm{d} u_{\mathrm{th}}}{\mathrm{d} x}= & -\frac{\alpha q(\xi)(1+\nu)}{\rho c k} \exp \left\{-\frac{V(x-\xi)}{2 k}\right\}\left[I_{0}\left\{\frac{V(x-\xi)}{2 k}\right\}-\right. \\
& \left.-I_{1}\left\{\frac{V(x-\xi)}{2 k}\right\}\right] H(x-\xi)
\end{aligned}
$$

where $H(x)$ is Heaviside's step function. 
The normal displacement gradient at $(x, 0)$ due to a distributed tensile traction $p(\xi)$ and a shear traction $t(\xi)$ in the range $-a<\xi<a$ is [2]

$\frac{\mathrm{d} u_{\mathrm{el}}}{\mathrm{d} x}=-\frac{1-\nu}{\mu \pi} \int_{-a}^{a} \frac{p(\xi) \mathrm{d} \xi}{x-\xi}-\frac{1-2 \nu}{2 \mu} t(x)$

where $\mu$ is the modulus of rigidity.

We assume that Coulomb's law of friction applies within the contact region and hence

$t(x)=-f p(x)$

Furthermore, the heat flux $q(x)$ into the half-plane is equal to the work done against friction and, since the punch is an insulator,

$q(x)=-f V p(x)$

Since the punch is rigid, the vertical displacement within the contact region $(-a<x<a)$ is constant and hence its derivative is zero. Substituting for $t(x)$ and $q(x)$ from eqns. (4) and (5) and using eqns. (2) and (3) we have

$$
\begin{aligned}
\frac{\mathrm{d} u}{\mathrm{~d} x}= & -\frac{1}{\pi} \int_{-a}^{a} \frac{p(\xi) \mathrm{d} \xi}{x-\xi}+\frac{\alpha f V(1+\nu) \mu}{k \rho c(1-\nu)} \int_{-a}^{a} p(\xi) \exp \left\{-\frac{V(x-\xi)}{2 k}\right\} \times \\
& \times\left[I_{0}\left\{\frac{V(x-\xi)}{2 k}\right\}-I_{1}\left\{\frac{V(x-\xi)}{2 k}\right\}\right] H(x-\xi) \mathrm{d} \xi+\frac{(1-2 \nu) f p(x)}{2(1-\nu)} \\
= & 0 \quad-a<x<a
\end{aligned}
$$

This equation may be simplified by introducing the dimensionless ratios

$\beta=\frac{1-2 \nu}{2(1-\nu)}$

$\mathrm{Pe}=V a / 2 k \quad$ (the Peclet number)

$J=\frac{2 \alpha \mu(1+\nu)}{\rho c(1-\nu)}$

$\widetilde{p}=p a / P$

We also normalize the interval of integration by setting

$\xi=a \omega$ and $x=a \eta$

so that

$$
\begin{aligned}
& \frac{1}{\pi} \int_{-1}^{1} \frac{\tilde{p}(\omega) \mathrm{d} \omega}{\omega-\eta}+J f \operatorname{Pe} \int_{-1}^{1} \tilde{p}(\omega) \exp \{-\operatorname{Pe}(\eta-\omega)\}\left[I_{0}\{\operatorname{Pe}(\eta-\omega)\}-\right. \\
& \left.-I_{1}\{\operatorname{Pe}(\eta-\omega)\}\right] H(\eta-\omega) \mathrm{d} \omega+f \beta \tilde{p}(\eta) \\
& \quad=0 \quad-1<\eta<1
\end{aligned}
$$


The Cauchy singular integral in eqn. (11) dominates the bounded Bessel function term and therefore determines the nature of the singularity in $\tilde{p}(\eta)$ at \pm 1 . Following ref. 3 , we introduce the appropriate unbounded fundamental solution by defining

$\tilde{p}(\omega)=\phi(\omega)(1-\omega)^{A-1}(1+\omega)^{-A}$

where

$\tan (\pi A)=-1 / f \beta \quad 0<A<1$

Once eqn. (11) has been solved, the temperature distribution at the surface may be found using the result for a moving line heat source from Carslaw and Jaeger [4], i.e.

$T=\frac{1}{\pi k \rho c} \int_{-a}^{a} q(\xi) \exp \left\{-\frac{V(x-\xi)}{2 k}\right\} K_{0}\left\{\frac{V(x-\xi)}{2 k}\right\} \mathrm{d} \xi$

\section{Discretization}

Equation (11) is a Cauchy singular integral equation of the second kind for which the appropriate quadrature is the Gauss-Jacobi technique described by Krenk [5].

Equation (11) reduces to the discretized form

$\sum_{i=1}^{n} A_{i}{ }^{n} \phi\left(\omega_{i}\right) K\left(\omega_{i}, \eta_{j}\right)=0 \quad j=1,2, \ldots, n-1$

where the integration points $\left(\omega_{i}\right)$ and the collocation points $\left(\eta_{j}\right)$ are given by the roots of the Jacobi polynomials [6]

$P_{n}{ }^{(A-1,-A)}\left(\omega_{i}\right)=0 \quad i=1,2, \ldots, n$

and

$P_{n-1}{ }^{(1-A, A)}\left(\eta_{j}\right)=0 \quad j=1,2, \ldots, n-1$

The kernel $K\left(\omega_{i}, \eta_{j}\right)$ includes both the integrals in eqn. (11) and $A_{i}{ }^{n}$ are the weight functions.

The extra equation is generated by ensuring overall equilibrium of the punch, i.e.

$-P=\int_{-a}^{a} p(\xi) \mathrm{d} \xi \quad$ or $\quad-1=\int_{-1}^{1} \tilde{p}(\omega) \mathrm{d} \omega$

In discretized form this is

$\sum_{i=1}^{n} A_{i}{ }^{n} \phi\left(\omega_{i}\right)=-1$ 
A set of $n$ simultaneous linear equations is formed from eqns. (14) and (18) for the unknowns $\phi\left(\omega_{i}\right)(i=1,2, \ldots, n)$. These are solved using a standard computer library routine.

Finally, the discretized form of eqn. (13) is

$\frac{\pi k \rho c}{f V P} T(\eta)=-\sum_{i=1}^{n} A_{i}{ }^{n} \phi\left(\omega_{i}\right) \exp \{-\operatorname{Pe}(\eta-\omega)\} K_{0}\{\operatorname{Pe}(\eta-\omega)\}$

\section{Limiting cases}

As the Peclet number is increased, the Green's function representing the moving heat source (eqn. (1)) becomes increasingly like a step function [1], so that in the limit $V \rightarrow \infty$ eqn. (1a) becomes

$u_{\mathrm{th}}=0 \quad x>\xi$

Equation (6) then takes the simplified form

$0=-\frac{1}{\pi} \int_{-a}^{a} \frac{P(\xi) \mathrm{d} \xi}{x-\xi}+(\beta+J) f p(x) \quad-a<x<a$

This equation can be solved exactly [5]. Its solution in normalized form is

$\tilde{p}(\omega)=\frac{1}{\pi} \sin \left\{\pi\left(A_{\infty}-1\right)\right\}(1-\omega)^{A_{\infty}-1}(1+\omega)^{-A_{\infty}} \quad-1<\omega<1$

where

$\tan \left(\pi A_{\infty}\right)=-\frac{1}{f(\beta+J)} \quad 0<A_{\infty}<1$

At the other extreme, if the punch slides at an infinitesimal speed, the Green's function (eqn. (1)) tends to a constant (rigid body) displacement which has no effect on the contact problem and we obtain an integral equation exactly similar to eqn. (21) except that the constant $J$ is omitted. The solution is again given by eqn. (22), except that the constant $A_{\infty}$ is replaced by $A_{0}$, where

$\tan \left(\pi A_{0}\right)=-\frac{1}{f \beta} \quad 0<A_{0}<1$

These two exact solutions bracket the numerically obtained intermediate solutions as shown in Fig. 2.

\section{Results}

Table 1 lists values of the dimensionless parameters $\beta$ and $J$ defined by eqns. (7) and (9) for a variety of metals and alloys. It is interesting to note 


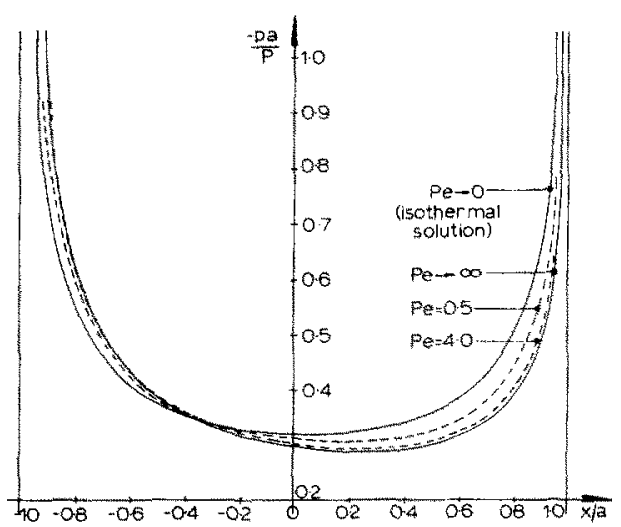

(a)

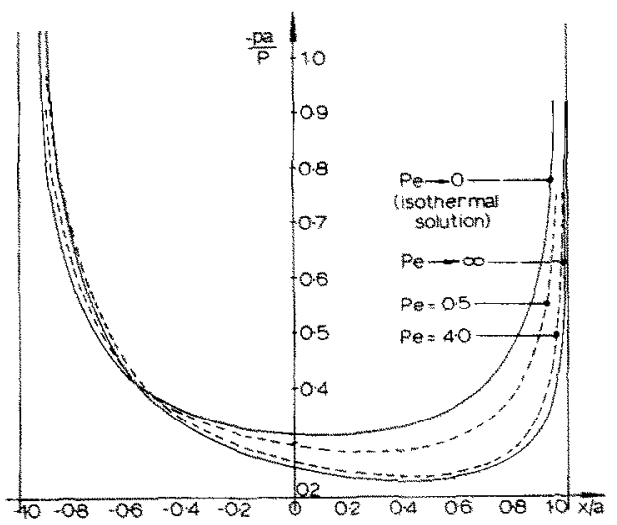

(b)

Fig. 2. Pressure distribution under the punch for the limiting cases $P e=0$ (isothermal) and $\mathrm{Pe} \rightarrow \infty$ (high speed) and for two intermediate values. In all cases, $J$ was taken to be unity and $\beta$ to be 0.25 : (a) $f=0.3 ;$ (b) $f=0.6$.

that the parameter $J$ is remarkably close to unity for most metals, despite considerable disparity in the contributory properties.

Numerical results were obtained for $J=1$ and $\beta=0.25$. Figures 2(a) and 2 (b) show the variation in pressure distribution with Peclet number for coefficients of friction $f=0.3$ and $f=0.6$ respectively. For very small Peclet number ( $\mathrm{Pe}=0.001$ ), convergence is obtained with $n>70$ and the numerical solution follows the exact isothermal solution very closely. As Pe increases, the number of points required for convergence also increases, since it is necessary to have a reasonable number of points in the region where the Green's function (eqn. (1)) has a significant slope. However, satisfactory results for $\mathrm{Pe}=4$ were obtained with $n=100$, and this curve is close to the theoretical limit for large Pe.

At high Peclet numbers the temperature attained is approximately constant over much of the contact, though with a sharp rise toward the 
TABLE 1

\begin{tabular}{lcclll}
\hline Metal & $\begin{array}{l}K \\
\left(\mathrm{~W} \mathrm{~m}^{-1}{ }^{\circ} \mathrm{C}^{-1}\right)\end{array}$ & $\begin{array}{l}k \\
\left(\times 10^{-5} \mathrm{~m}^{2} \mathrm{~s}^{-1}\right)\end{array}$ & & & \\
& & 8.628 & 0.34 & 0.2727 & 1.022 \\
$\mathrm{Al}$ & 205 & 3.838 & 0.35 & 0.2307 & 1.010 \\
$\mathrm{Brass}$ & 120 & 11.454 & 0.35 & 0.2307 & 0.954 \\
$\mathrm{Cu}$ & 390 & 11.08 & 0.42 & 0.1379 & 0.686 \\
$\mathrm{Au}$ & 310 & 2.201 & 0.29 & 0.2957 & 0.960 \\
Iron (wrought) & 76 & 2.458 & 0.44 & 0.1071 & 0.629 \\
$\mathrm{~Pb}$ & 35 & 1.415 & 0.30 & 0.2857 & 0.937 \\
$\mathrm{Mild}$ steel & 50 & 2.302 & 0.31 & 0.2753 & 0.987 \\
$\mathrm{Ni}$ & 91 & 2.567 & 0.38 & 0.1935 & 0.909 \\
$\mathrm{Pt}$ & 69 & 17.16 & 0.37 & 0.2063 & 0.950 \\
$\mathrm{Ag}$ & 418 & 3.914 & 0.36 & 0.2188 & 1.016 \\
$\mathrm{Sn}$ & 64 & 7.572 & 0.38 & 0.1935 & 1.118 \\
$\mathrm{~W}$ & 190 & 4.145 & 0.35 & 0.2308 & 1.700 \\
$\mathrm{Zn}$ & 113 & & & & \\
\hline
\end{tabular}

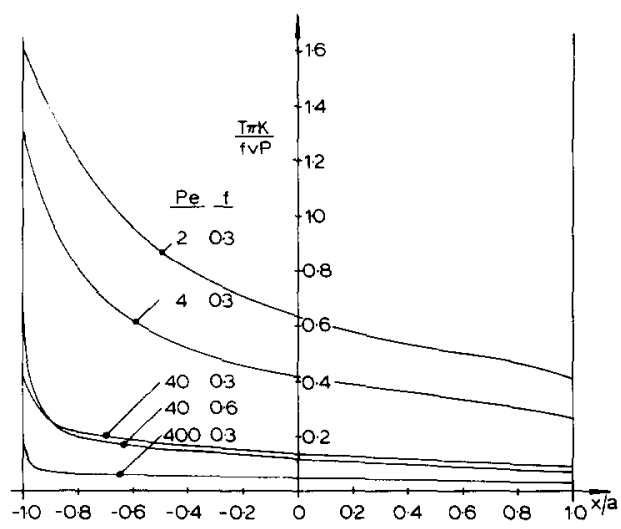

Fig. 3. Temperature distribution under the punch for various values of Pe and $f$. In all cases, $J$ was taken to be unity and $\beta$ to be 0.25 .

trailing edge (Fig. 3). For lower Peclet numbers, where the rate of diffusion is more comparable with the sliding speed, a significant temperature rise is seen over the whole of the contact patch. These results are to be expected from physical intuition; it is clear that even if heat were generated uniformly over the contact the trailing portion will be warmer and hence will expand vertically more than the leading part of the contact. This in turn increases the contact pressure and hence leads to the generation of more heat. The analysis presented refers to the steady state, and Fig. 2 confirms an increase in contact pressure toward the trailing edge, though this is, to some extent, masked by the singular nature of the pressure distribution inherent in all rigid punch contact problems. Nevertheless, the solution presented shows clearly the application of steady state thermal Green's functions [1] to contact problems, enables us to assess the contribution of thermal distor- 
tion quantitatively and provides a closed-form bounding solution at high sliding speeds.

\section{Acknowledgments}

The authors gratefully acknowledge support for this work from the English Speaking Union (D.A.H.) and from the U.S. Army under Contract DANG 29-82-K-0001 (J.R.B.).

\section{References}

1 J. R. Barber, Thermoelastic displacements and stresses due to a heat source moving over the surface of a half-plane, ASME Prepr. 84-WA/APM-17, 1984 (American Society of Mechanical Engineers); J. Appl. Mech., 51 (3) (1984) $636-640$.

2 N. I. Muskhelishvili, Some Basic Problems of the Mathematical Theory of Elasticity (transl. J. R. M. Radok), Noordhoff, Groningen, 1953, Chapter 19, pp. 475 - 477.

3 N. I. Muskhelishvili, Singular Integral Equations, Noordhoff, Groningen, 2nd edn., 1953 , pp. $299-305$.

4 H. S. Carslaw and J. C. Jaeger, Conduction of Heat in Solids, Clarendon, Oxford, 2nd edn., 1959, p. 267.

$5 \mathrm{~S}$. Krenk, On quadrature formulas for singular integral equations of the first and second kind, Q. Appl. Math., 33 (1975) 225 - 232.

6 A. H. Stroud and D. Secrest, Gaussian Quadrature Formulas, Prentice-Hall, Englewood Cliffs, NJ, 1966. 